\title{
CFAl-Plus: Adding cognitive frailty as a new domain to the comprehensive frailty assessment instrument
}

\author{
Ellen Elisa De Roeck ${ }^{1,2}$ (D) | Sarah Dury ${ }^{3,4}$ | Nico De Witte ${ }^{3,5}$ | Liesbeth De Donder ${ }^{3}$ | \\ Maria Bjerke $^{2}$ | Peter Paul De Deyn ${ }^{2,6}$ | Sebastiaan Engelborghs ${ }^{2,6}$ | Eva Dierckx ${ }^{1}$
}

\author{
${ }^{1}$ Department of Clinical and Lifespan \\ Psychology, Vrije Universiteit Brussel, \\ Brussels, Belgium \\ ${ }^{2}$ Laboratory of Neurochemistry and Behavior, \\ University of Antwerp, Antwerp, Belgium \\ ${ }^{3}$ Department of Educational Sciences, Vrije \\ Universiteit Brussel, Brussels, Belgium \\ ${ }^{4}$ Research Foundation Flanders (FWO), \\ Egmontstraat 5, 1000 Brussels, Belgium \\ ${ }^{5}$ Faculty of Education, Health and Social \\ Work, University College Ghent, Ghent, \\ Belgium \\ ${ }^{6}$ Department of Neurology and Memory \\ Clinic, Hospital Network Antwerp (ZNA) Hoge \\ Beuken and Middelheim, Antwerp, Belgium

\section{Correspondence} \\ E. Dierckx, Department of Clinical and \\ Lifespan Psychology, Vrije Universiteit Brussel, \\ Brussels, Belgium. \\ Email: eva.dierckx@vub.be \\ Funding information \\ Flanders Impulse Program on Networks for \\ Dementia Research; Research Foundation \\ Flanders; Agency for Innovation by Science \\ and Technology, Grant/Award Number: IWT- \\ 140027-SBO; Flanders Impulse Program on \\ Networks for Dementia Research (VIND); \\ University of Antwerp Research Fund
}

Objectives: Cognitive frailty is characterized by the presence of cognitive impairment in exclusion of dementia. In line with other frailty domains, cognitive frailty is associated with negative outcomes. The Comprehensive Frailty Assessment Instrument (CFAI) measures 4 domains of frailty, namely physical, psychological, social, and environmental frailty. The absence of cognitive frailty is a limitation.

Method: An expert panel selected 6 questions from the Informant Questionnaire on Cognitive Decline that were, together with the CFAl and the Montreal cognitive assessment administered to 355 older community dwelling adults (mean age $=77$ ).

Results: After multivariate analysis, 2 questions were excluded. All the questions from the original CFAl were implemented in a principal component analysis together with the 4 cognitive questions, showing that the 4 cognitive questions all load on 1 factor, representing the cognitive domain of frailty. By adding the cognitive domain to the CFAl, the reliability of the adapted CFAI (CFAI-Plus), remains good (Cronbach's alpha: .767).

Conclusions: This study showed that cognitive frailty can be added to the CFAl without affecting its good psychometric properties. In the future, the CFAI-Plus needs to be validated in an independent cohort, and the interaction with the other frailty domains needs to be studied.

\section{KEYWORDS}

CFAl, cognition, cognitive impairment, frailty

\section{1 | INTRODUCTION}

With the growth of the older population worldwide, ${ }^{1}$ frailty has become an increasingly relevant concept. There is a growing tendency to view frailty from a multidimensional perspective. ${ }^{2-5}$ The multidimensional approaches have defined frailty as a dynamic state affecting 1 or more domains of human functioning, which is caused by a range of variables and which increases the risk of adverse outcomes. ${ }^{5}$ Within this multidimensional approach, the most described frailty domains are physical, psychological, social, and recently also environmental frailty. $^{2}$ Conversely, 1 domain of human functioning remained

Sponsor: This work was supported by the Agency for Innovation by Science and Technology (grant: IWT-140027-SBO) underexposed, namely cognitive functioning. This is surprising as impaired cognitive functioning can have a significant impact on the different frailty domains and can as well limit the possible therapeutic options. $^{6,7}$ Therefore, cognitive frailty gained attention as a frailty domain. ${ }^{8-11}$ Although some researchers describe cognitive frailty as the presence of both physical and cognitive impairments, ${ }^{9}$ consensus is growing that cognitive frailty is an independent frailty domain. In addition, Buchman and Bennet (2013) ${ }^{8}$ argue that it is important to assess individual domains to get more profound knowledge about specific limitations. Recently, Panza and colleagues ${ }^{12}$ suggested classifying cognitive frailty into a reversible and a potentially reversible form. Hereby, the reversible form is comparable to Subjective Cognitive Impairment and can be seen as precursor of potentially reversible cognitive frailty. The potentially reversible form is related to mild 
cognitive impairment $(\mathrm{MCl})$, which means there has to be objective cognitive impairment. For this study, we will concentrate on the reversible form. Hence, how to operationalize cognitive frailty appears one of the most essential issues. 8,13

The Comprehensive Frailty Assessment Instrument (CFAI) is a self-reported questionnaire with good psychometric properties that is developed to measure multiple domains of frailty from a holistic approach. $^{2}$ The CFAI is administered to community dwelling older people (60 years and older) with the goal to detect self-perceived frailty. The instrument is developed and used as a screening tool. However, the CFAl only measures the aforementioned 4 frailty domains. The absence of cognitive frailty can be seen as a limitation of this instrument. When the original CFAl was developed, some important criteria were used, like emphasis on the experience of older persons themselves. Accordingly, if we want to add a cognitive section to the CFAl, self-reported questions on cognitive frailty should be incorporated that explore subjective cognitive complaints. Mitchell $(2014)^{14}$ already established that subjective cognitive complaints (ie, cognitive frailty) could be a precursor of actual cognitive decline based on a meta-analysis of longitudinal studies. Hence, for clinical practice as well as for longitudinal studies, it is worth to follow up people with subjective cognitive complaints as they may be at risk for cognitive decline. Moreover, subjective cognitive complaints are associated with negative (health) outcomes. For example, with the LEILA75+ study, researchers showed an association between subjective cognitive complaints and mortality. ${ }^{15}$ In their study, non-demented older adults (75+) with subjective cognitive complaints had an increased mortality risk in comparison with subjects without cognitive complaints.

Another valuable reason to recognize the importance of subjective cognitive complaints can be the negative impact it can have on someone's quality of life. For example, Zuniga and colleagues $(2016)^{16}$ found a negative association between subjective memory complaints and well-being in older adults. Moreover, researchers showed an association between cognitive complaints, depression, and lower quality of life. ${ }^{17}$

In sum, the higher risk of cognitive decline and the negative impact on well-being result in a need to acknowledge cognitive frailty as a serious issue in the life of older individuals. To avoid these negative outcomes, a timely and accurate detection of cognitive frailty is essential. The goal of this study is to add a cognitive frailty domain to the CFAI, resulting in the CFAI-Plus.

\section{2 | METHODS}

\section{1 | Participants}

For this study, data of 355 community-dwelling participants aged between 62 and 101, all living in the northern part of Belgium, Flanders was used. The exclusion criteria were as follows: (1) a diagnosis of cognitive impairment $(\mathrm{MCl}$ or dementia) given by a doctor (specialist or general practitioner), (2) the intake of a medicine to treat Alzheimer's disease (for example a cholinesterase inhibitor), (3) no or low knowledge of the Dutch language, and (4) illiteracy. Psychology students from the Vrije Universiteit Brussel gathered all data after an

\section{Key points}

- Cognitive frailty can be added to the original CFAl, resulting in the CFAI-plus.

- The CFAl-plus has good psychometric qualities.

- CFAl-Plus is an easy to administer tool for the identification of individuals with multidimensional frailty in the general older population.

intensive training to administer the different tests and questionnaires. The study was conducted between October 2015 and April 2016. All participants gave written informed consent. The ethical committee of human sciences of the Vrije Universiteit Brussel approved the research protocol.

\section{2 | Measurements}

\subsection{1 | Montreal cognitive assessment (MoCA)}

The MoCA is a brief cognitive screening tool designed to detect $\mathrm{MCl}$ or mild dementia. ${ }^{18}$ The MoCA examines multiple domains of cognitive functioning, such as short-term memory, visuospatial ability, executive function, attention, language, and orientation in time and place. The score ranges from 0 to 30 , where higher scores indicate better cognition. In order to correct for education effects, 1 point was added to the total score for participants with less than 12 years of education. ${ }^{18}$

Different studies have shown that the recommended cut-off score of 26 leads to a lot of false positives. ${ }^{19,20}$ Consequently, we added 2 different cut-off points of 24 and of 21 in our analysis. A series of validation studies in western countries have shown that a score under 24 is probably indicative for mild cognitive problems, ${ }^{19,20}$ whereas a score of 21 or less is indicating cognitive impairment. ${ }^{21}$ In addition, the use of 3 different cut-offs gives us the opportunity to differentiate between no (MoCA $\geq 26$ ), mild ( $\mathrm{MoCA}<24)$, and severe (MoCA<21) cognitive problems.

\subsection{2 | The Comprehensive Frailty Assessment Instrument}

The CFAl is well described by De Witte. ${ }^{2}$ The CFAl measures 4 domains of frailty (physical, psychological, social, and environmental frailty) each ranging from 0 to 25 , with higher scores indicating a higher risk of frailty on that domain. Both the psychological and social domain of frailty consists of 2 subdomains, each ranging from 0 to 12.5. The psychological frailty domain includes mood-disorders and emotional Ioneliness. Social frailty domain comprises social loneliness and social support network. For the physical frailty domain, older adults are invited to report limitations in physical activities due to health problems. For the environmental domain, questions are asked about their actual housing conditions. All answers, except for the social support network, are given on Likert scales from 0 (not applicable at all/occurring not at all) to maximum 4 (completely applicable). The social support network subdomain is answered with a dummy (yes = can get help from, no = no help from). 
In order to certify that each of the 4 domains of the original CFAI contributes equally ( $25 \%$ each) to the total score, all domain scores are rescaled on 25 . Thus, a formula is used:

$$
\frac{(\text { sum items }) \times 25}{(\text { sum maximum scores on each item })}=\text { domain score } .
$$

Finally, the total score of the CFAl is calculated by summing up the 4 domain scores, resulting in a score from 0 to 100. A higher score indicates higher risk of frailty. A version of the CFAl-plus is available online (Supporting Information).

\subsubsection{Selection of the cognitive frailty questions}

An expert panel consisting of 2 neurologists specialized in dementia (SE and PPDD), a psychologist specialized in neuropsychology and dementia (ED), and 3 adult educational scientists specialized in social gerontology (LDD, SD, NDW) selected 6 questions from the Informant Questionnaire on Cognitive Decline in the Elderly Dutch version $(\text { IQCODE-N })^{22,23}$ based on what they experienced as most typical of the cognitive frailty domain. The IQCODE-N is a 16-item self-reported questionnaire designed to assess cognitive decline and dementia in older people and correlates with a wide range of cognitive tests. ${ }^{24}$ The following 6 questions were selected:

1. I have trouble with remembering things that have happened recently.

2. I have trouble with recalling a conversation a few days later.

3. I experience difficulties with learning new things in general.

4. I have trouble with learning to use new machines around the house.

5. I experience difficulties with handling financial matters, eg, the pension, dealing with the bank.

6. I have trouble with following a story in a book or on TV.

In line with other questions from the CFAI, participants rated each question on a 5-point scale where 0 represents "totally disagree", 1 = "disagree", 2 = "neither agree / nor disagree", 3 = "agree", and 4 = "totally agree".

\section{3 | Procedure}

After written informed consent was obtained, basic demographic questions were asked (age, education, etc.), and the MoCA was administered according to the guidelines. Feedback on objective test performance was not given to not influence subjective ratings of cognition. Thereafter, the original CFAl and the 6 additional questions were handed to the participant. The participant was encouraged to complete the questionnaire without any further direction from the administrator.

\section{4 | Statistics}

SPSS 23 (version 24; SPSS, Inc, Chicago, III) and SIMCA P+ software (v 13.0, Umetrics, Umeå, Sweden) were used.

First, exploratory analyses of the skewness and kurtosis of the most important variables were done to check for normal distribution. Next to that, baseline characteristics were calculated, such as mean age, MoCA, and CFAl scores.
Second, factor analyses (with SIMCA P+ software) were performed to select the best items measuring the cognitive frailty domain. To explore which of the 6 cognitive frailty questions best predicts cognitive frailty, the answers on the 6 questions together with the MoCA score groups were entered into an orthogonal projection to latent structures discriminant analysis (OPLS-DA). The OPLS-DA algorithm discovers the projection direction, the score vector, that gives the largest covariance between the variables and the pre-defined classes (ie, MoCA under and above the cut-off) and that maximizes the separation between the 2 classes. Based on this analysis, questions were selected that had the highest contribution on the projection and that best contributed to the discrimination between the classes. Variable Influence on the Projection plot (VIP plot) visually presents the contribution, see Figure 1. The height of the VIP bar indicates the amount of contribution of the variable on the model. The VIP plot also offers a $95 \%$ confidence interval $(\mathrm{Cl})$ for the contribution of each variable.
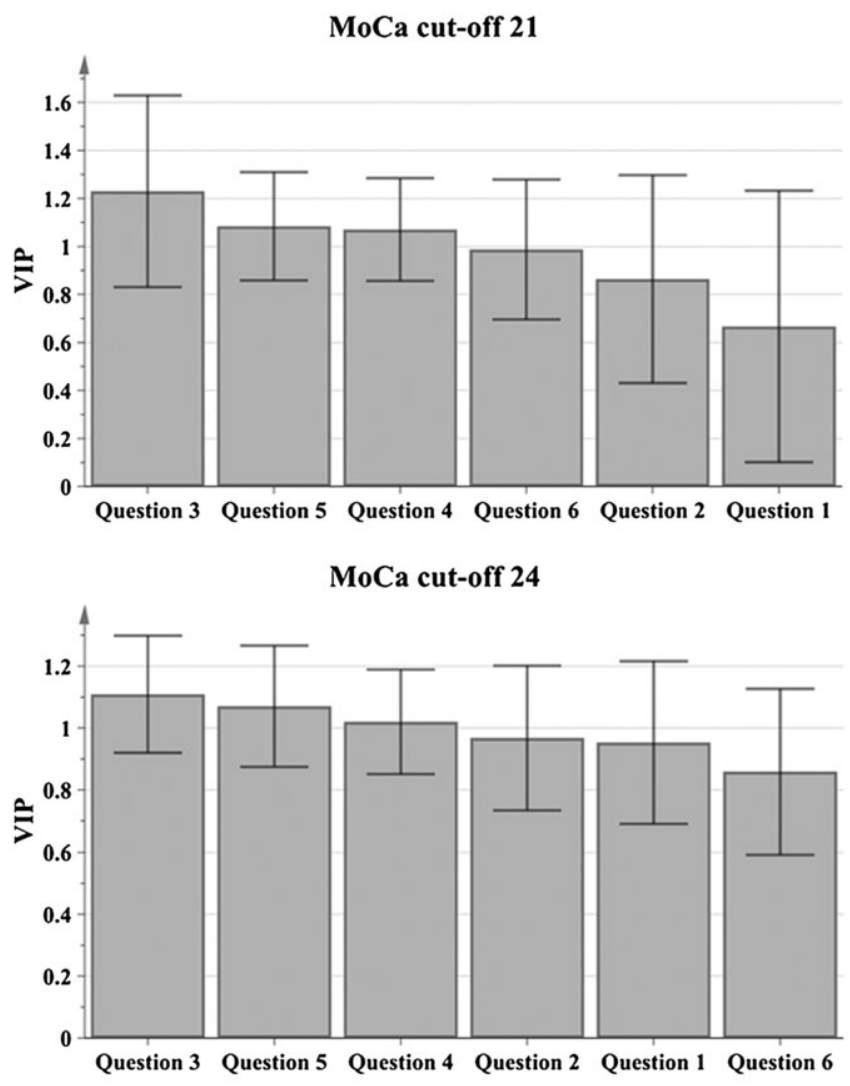

MoCa cut-off 26

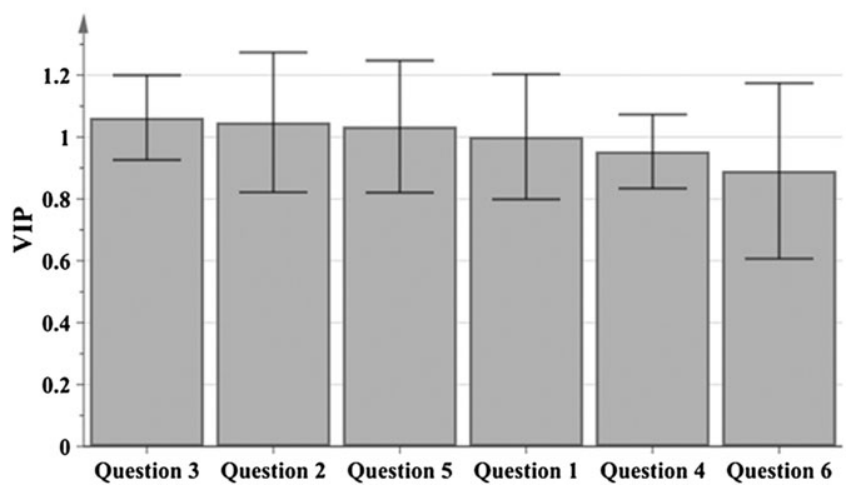

FIGURE 1 Represent the variable influence on the projection plot (VIP plots) of the 6 questions for the 3 different cut-off points of the MoCA 
In addition, in SPSS, areas under the curve (AUC) were calculated for different combinations of questions to see the diagnostic value for cognitive impairment (as measured with the MoCA). Based on the OPLS-DA in combination with the AUC the most informative questions were selected. The combination of the selected questions was defined as the cognitive frailty domain. In order, to see how the cognitive questions related to the other questions from the CFAI, a principal component analysis (PCA) was conducted. In order to enhance interpretability, oblique (oblimin) rotations were applied. Regarding the number of patterns to retain, the Kaiser criterion was used. As the 10 questions about the social network are answered on another scale (yes or no) than the other questions (Likert scale), they were not included in this analysis.

In addition, to measure the internal consistencies of the cognitive frailty questions and for the CFAl in combination with the questions, Cronbach's alphas were computed together with the inter-item correlation of the questions. To gain insight into the concurrent validity of cognitive frailty, the correlation between cognitive frailty and MoCA was further studied through correlation (Pearson) and linear regression analyses.

Finally, in line with the other CFAl domains, ${ }^{25}$ the cut-off for cognitive frailty was calculated with a 2-step cluster analysis. For this analysis, the respondents' scores on cognitive frailty are automatically hierarchically clustered using Schwarz's Bayesian Criterion and Log-likelihood for distance measure.

\section{3 | RESULTS}

\subsection{Exploratory analyses and participant baseline characteristics}

Exploratory analyses showed that for age, MoCA score, total CFAl score, and cognitive frailty score, the skewness ranged from 0.062 to 0.581 and the kurtosis from -0.372 to -0.916 . Thus, there was no indication of a strong deviation from normality. Of the 355 selected participants, 353 completed all tests and questionnaires. Two were excluded due to missing values. Table 1 gives an overview of the mean age, MoCA, and CFAl scores.

\subsection{Selection of the questions}

To select the best questions, OPLS-DA analyses were carried out. Figure 1 gives an overview of the VIP plots for every cut-off point

TABLE 1 Baseline characteristics: the mean (M) and standard deviation (SD) of the MoCA and the original CFAl scores for all participants

\begin{tabular}{lc} 
& Total group (M + SD) \\
\hline $\mathrm{n}$ & 353 \\
\hline Gender (male/female) & $159 / 194$ \\
\hline Age & $77.69(8.33)$ \\
\hline MoCA & $25.13(3.20)$ \\
\hline CFAl physical & $9.10(8.43)$ \\
\hline CFAl psychological & $4.44(4.50)$ \\
CFAl social & $9.82(4.98)$ \\
\hline CFAl environmental & $2.60(3.54)$ \\
\hline CFAl total & $25.95(13.57)$
\end{tabular}

of the MoCA. For all of the cut-off points, question three is selected as the most important question. This is followed by question five. Looking at the other questions, these are less stable predictors for the models based on the different MoCA cut-offs. Thereafter, the AUC were calculated if each question or if combinations of questions were deleted. The results of the AUC for each MoCA cut-off are presented in Table 2. As the best grouping of questions appeared a combination of question 1, 3, 5, and 6, these questions were chosen to represent the cognitive frailty domain, which was further tested below.

\section{3 | Different domains of the CFAl}

To see how the cognitive questions related to the other questions in the CFAI, a principal component analysis was conducted. As expected, the analysis suggested extracting the 5 known components. All 5 components were meaningful and represented the different domains of the CFAl. Component loadings were very good for the cognitive (.712-.654), psychological (.779-.515), physical (.830-.657), and social domain (.901-.856) (Abdi and Williams 2010). The loadings of the fifth component, environmental frailty, were reasonable (.761-.297). The only question with a low loading in this domain was "I do not like the neighborhood".

So, in sum, the PCA shows that the 4 domains already established in the CFAl were represented again. Hence, the cognitive questions together formed 1 principal (cognitive) component, establishing the fifth domain of the CFAI-Plus.

\subsection{Reliability of cognitive frailty and the CFAI-Plus}

The cognitive frailty domain was found to have reasonable internal reliability (Cronbach's alpha $=.665$ ). The CFAl without the items of the cognitive domain had a good internal reliability of alpha $=.724$. By combining the CFAl with the items of the cognitive domain, the reliability elevated to alpha $=.767$.

TABLE 2 Overview of the area under the curve (AUC) scores for each MoCA cut-off score, separately for each (group of) excluded question(s)

\begin{tabular}{|lccc|} 
& $\begin{array}{l}\text { MoCA } \\
\text { cut-off 21 } \\
\text { AUC }\end{array}$ & $\begin{array}{l}\text { MoCA } \\
\text { cut-off 24 } \\
\text { AUC }\end{array}$ & $\begin{array}{l}\text { MoCA } \\
\text { cut-off 26 } \\
\text { AUC }\end{array}$ \\
\hline All 6 questions & .627 & .652 & .668 \\
\hline 1 excluded & .643 & .651 & .666 \\
\hline 2 excluded & .636 & .663 & .669 \\
\hline 4 excluded & .620 & .649 & .672 \\
\hline 6 excluded & .620 & .651 & .667 \\
\hline 4 and 6 excluded & .610 & .648 & .672 \\
\hline 4 and 2 excluded & .633 & .666 & .677 \\
\hline 1 and 4 excluded & .637 & .647 & .671 \\
\hline 1 and 6 excluded & .637 & .648 & .662 \\
\hline 2 and 6 excluded & .627 & .663 & .666 \\
\hline 2 , 4, and 6 excluded & .621 & .667 & .676 \\
\hline 1,4 , and 6 excluded & .632 & .645 & .667 \\
\hline
\end{tabular}




\subsection{Concurrent validity of the cognitive frailty domain}

There was a significant $(P<.001)$ negative correlation between the MoCA and cognitive frailty $(r=-.318)$ indicating that more subjective cognitive complaints are associated with lower scores on the MoCA (ie, more $\mathrm{MCl}$ ). If entered in a linear regression model, the cognitive frailty questions were significantly able to predict scores on the MoCA $(R=.319, F(1,351)=39,54, P<.001)$. However, the explained variance of $10.1 \%$ is weak. $^{26}$

\subsection{Cut-off for the cognitive frailty domain}

Before being able to perform the 2-step cluster analysis, the data were transformed. In the original CFAl, each domain represents an equal weight to the total CFAl, namely 25 points of the total 100 points. As a result of the addition of the cognitive frailty domain, which we also transformed to 25 points, the new total is 125 points.

The 2-step cluster analysis revealed 3 natural groupings (or clusters) within our data set for cognitive frailty that are in line with the other domains: (1) "not-to-low" cognitively frail

group, (2) "low-to-medium", and (3) "medium-to-high" cognitively frail group. Table 3 gives an overview of the different groups and their cut-offs and scores on the other frailty domains.

\section{4 | DISCUSSION}

The aim of the present study was to add a cognitive domain to the CFAl in order to have a more comprehensive insight on multidimensional frailty in an aging population. From the 6 eligible questions, we were able to select 4 questions to develop a cognitive frailty domain. When entered in a factor analysis, these 4 items formed 1 cognitive component. Moreover and crucial is the reconfirmation that the items from the other domains remain in the original 4 components. Our results indicate that the reliability of the CFAI-Plus was comparable to that of the original version of the CFAI. The internal reliability of the CFAl-Plus was even higher as compared with the original CFAl. Besides, there was a significant, though rather weak, correlation between the new cognitive domain and scores of the MoCA, indicating that higher scores on cognitive frailty are associated with cognitive impairment. This result is in line with previous research as a short 7-item version of IQCODE successfully discriminated between $\mathrm{MCl}$ / $A D$ patients and healthy controls. ${ }^{24}$ In conclusion, the ability to measure cognitive frailty with the CFAI-Plus may have an added value for further research as well as for (clinical) practice and policy purposes.

\subsection{Identification of cognitively frail older adults}

In our total sample, $26.5 \%$ of the subjects scored mild to high on the cognitive frailty domain. This number is comparable with other studies showing that subjective complaints are common in older people. ${ }^{14,27,28}$ It is noteworthy to report that persons with cognitive complaints were significantly older $(M=81.35, \mathrm{SD}=7.45)$ than persons without complaints $(M=73.32, S D=6.96)(t(191)=7.61, P=.000)$. This is in line with a study of Montejo and colleagues. (2011) ${ }^{27}$ who found that subjective cognitive complaints increase with age. Indeed, in people with ages ranging between 65 and 69 years, 24\% had subjective cognitive complaints whereas in people of more than 90 years, $57 \%$ had subjective complaints.

Previous studies indicated that the prevalence of dementia in people aged 60 years and over is $5 \%$ to $7 \%$ and that these prevalence rates increase with age. ${ }^{29}$ Thus, it is not unexpected that in our study $15 \%$ scored 21 or less on the MoCA, indicating that some of these participants probably suffer from $\mathrm{MCl}$ or dementia. Although the correlation between the MoCA and CFAl cognitive domain was rather weak (and as a consequence also the explained variance of our regression analyses), $88.9 \%$ of the people who probably suffer from $\mathrm{MCl}$ or dementia (MoCA <21) reported on the CFAI-Plus that they were cognitive frail or prefrail. This indicates that a large proportion of people with cognitive problems can report this through the cognitive frailty domain.

From the 132 participants with a MoCA score above 26 (probably without cognitive impairment), also $16 \%$ reported to be cognitive frail. Mitchell (2014) $)^{14}$ concluded from a meta-analysis of longitudinal studies that after 4 years $24.4 \%$ of older individuals with subjective memory complaints developed $\mathrm{MCl}$, while $10.9 \%$ converted to dementia, compared with only $4.6 \%$ in older adults without subjective memory complaints. This may indicate that this group with complaints has a higher risk to develop a cognitive impairment in the future.

In conclusion, the higher risk of cognitive decline for people with subjective complaints and the option to detect people with actual

TABLE 3 The mean and standard deviation of age, MoCA scores, and CFAl domain scores for the 3 subgroups based on a 2-step cluster analysis

\begin{tabular}{lccc} 
& $\begin{array}{l}\text { Non-Low Cognitively Frail } \\
\text { (Mean + SD) }\end{array}$ & $\begin{array}{l}\text { Low-Mild Cognitively Frail } \\
\text { (Mean + SD) }\end{array}$ & $\begin{array}{c}\text { Mild-High Cognitively Frail } \\
\text { (Mean + SD) }\end{array}$ \\
\hline$n$ & 122 & 137 & 94 \\
\hline Gender (male/female) & $53 / 69$ & $66 / 71$ & $81.66(7.78)$ \\
\hline Age & $73.52(7.09)$ & $78.69(8.12)$ & $24.11(3.02)$ \\
\hline MoCA & $26.45(2.50)$ & $24.65(3.48)$ & $10.94-25$ \\
\hline Range for cognitive frailty & $0-3.13$ & $4.69-9.38$ & $14.10(3.08)$ \\
CFAl cognitive & $1.18(1.28)$ & $6.75(1.79)$ & $12.83(8.19)$ \\
\hline CFAl physical & $5.48(6.88)$ & $9.76(8.62)$ & $6.98(4.48)$ \\
CFAl psychological & $2.65(3.76)$ & $4.29(4.34)$ & $9.77(4.39)$ \\
\hline CFAl social & $9.79(5.25)$ & $9.88(5.15)$ & $3.22(3.93)$ \\
CFAl environmental & $1.49(3.03)$ & $3.16(3.46)$ & $46.90(12.60)$ \\
CFAl total & $20.58(11.40)$ & $33.84(14.04)$ & \\
\hline
\end{tabular}


cognitive impairment make it important to add cognitive frailty as a domain to existing multidimensional screening instruments.

\section{2 | Strengths and limitations}

The new cognitive frailty domain of the CFAI-Plus has several advantages. It is short, easy to administer, and enables comparison of cognitive frailty with the other frailty domains. Moreover, the CFAI-Plus is the first multidimensional frailty instrument that takes into account 5 frailty domains. However, some possible limitations require further discussion. For example, the CFAI-Plus may not be a reliable cognitive screening instrument in subjects with more severe cognitive impairments. Hence, we noticed that from the people with a MoCA score below $21,11 \%$ did not report cognitive frailty. This result might be explained by the unawareness of the existence of cognitive deficits by at least part of the people that have (prodromal) dementia. ${ }^{30}$ Another restraint of the CFAI-Plus arises from the study population that was used to develop it. For this study, as well as for the original CFAl study, a community-dwelling sample of older people was used. The instrument is also originally developed to screen for frailty in people still living at home. Therefore, the psychometric properties of the CFAl-Plus for other populations (eg, institutionalized or hospitalized older people) are unclear. Consequently, it is advised to only use the CFAl-Plus for community-dwelling older people. Another limitation that needs to be addressed is the use of the MoCA (which is a cognitive screening test) to detect cognitive impairment instead of a full neuropsychological assessment. On the other hand, both MoCA and CFAI-Plus are screening instruments; from this point of view, it makes sense to compare them in one and the same population. Another limitation is the presented cut-off scores for the cognitive frailty domain. The cut-off for cognitive frailty is calculated in the same way as the cut-offs for the other original CFAI-domains. However, while the original domains were validated with very large sample sizes ( $n=33629$ ), ${ }^{25}$ in this study only a group of 353 participants was included. In order to replicate these findings and (if needed adapt) the cut-off, the CFAI-Plus will be added to the Belgian Aging Studies. Consequently, the findings will be replicated in an independent and larger cohort. A last shortcoming of this study is that it is not discussing the predictive value of the CFAI-Plus for negative (health) outcomes. Especially, for clinical practice, it would be helpful to see which frailty domains, or combinations of frailty domains, are associated with specific negative outcomes. This is an important topic for future studies.

\subsection{Clinical value}

In line with the CFAl, the CFAl-plus is a fast, easy, and inexpensive screening tool for assessing 5 different domains of frailty in community dwelling older people. By adding cognitive frailty to the CFAI, community health care providers are able to gain a more holistic view of the older person. Moreover, cognitive impairments can have a significant effect on the possible therapeutic options, therefore making it important to measure this as a separate domain. The administration of the CFAl can help health care providers to choose appropriate further assessments and interventions to prevent negative outcomes such as low quality of life or institutionalization.

\section{5 | CONCLUSIONS}

In conclusion, this study showed that cognitive frailty can be measured with 4 self-reported questions from the IQCODE and that these items can be added to the CFAI to create a cognitive frailty domain. Furthermore, the CFAI-Plus is a simple tool for the identification of individuals with multidimensional frailty in the general older population. As all the different frailty domains have a significant impact on people's lives and on their use of care and support the CFAI-Plus can be used as a first step for screening programs. For future research, special attention should go to (1) the replication of our results in an independent and larger sample and (2) the interaction between cognitive frailty and the other frailty domains.

\section{FUNDING}

This work was supported by the University of Antwerp Research Fund, the Flanders Impulse Program on Networks for Dementia Research (VIND), the Agency for Innovation by Science and Technology (IWT, www.iwt.be), and the Research Foundation Flanders (FWO, www. fwo.be).

\section{ACKNOWLEDGEMENTS}

The authors especially thank the D-SCOPE consortium. The research of the D-SCOPE consortium (IWT-140027-SBO) is composed of researchers from University of Antwerp, Vrije Universiteit Brussel, College University Ghent, Catholic University of Leuven, Belgium, and Maastricht University, the Netherlands: PP. De Deyn, L. De Donder, J. De Lepeleire, EE. De Roeck, N. De Witte, E. Dierckx, D. Duppen, S. Dury, S. Engelborghs, B. Fret, L. Hoeyberghs, T. Kardol, GIJM. Kempen, D. Lambotte, B. Schoenmakers, JMGA. Schols, A-S. Smetcoren, M. Van der Elst, A.van der Vorst, D. Verté, GAR. Zijlstra.

\section{CONFLICT OF INTEREST}

None declared

\section{ORCID}

Ellen Elisa De Roeck (i) http://orcid.org/0000-0002-6566-6562

\section{REFERENCES}

1. Global AGE Watch 2015. Global rankings table http://www.helpage. org/global-agewatch/ Published 2015. Accessed May 222017.

2. De Witte N, Gobbens R, De Donder L, et al. The comprehensive frailty assessment instrument: development, validity and reliability. Geriatr Nurs. 2013;34(4):274-281. https://doi.org/10.1016/j.gerinurse.2013.0 3.002

3. Dury S, De Roeck E, Duppen D, et al. Identifying frailty risk profiles of home-dwelling older people: focus on sociodemographic and socioeconomic characteristics. Aging Ment Health. 2016;21(10):10311039. https://doi.org/10.1080/13607863.2016.1193120

4. Grenier A. Constructions of frailty in the English language, care practice and the lived experience. Ageing Soc. 2007;27(03):425-445. https://doi. org/10.1017/S0144686X06005782

5. Gobbens RJ, Luijkx KG, Wijnen-Sponselee MT, Schols JM. Towards an integral conceptual model of frailty. J Nutr Health Aging. 2010;14(3): 175-181. https://doi.org/10.1007/s12603-010-0045-6 
6. Arts $\mathrm{MH}$, Collard RM, Comijs HC, et al. Physical frailty and cognitive functioning in depressed older adults: findings from the NESDO study. JAMDA. 2016;17(1):36-43. https://doi.org/10.1016/j.jamda.2015.0 7.016

7. Halil M, Kizilarslanoglu MC, Kuyumcu ME, et al. Cognitive aspects of frailty: mechanisms behind the link between frailty and cognitive impairment. J Nutr Health Aging. 2015;19(3):276-283. https://doi.org/ 10.1007/s12603-014-0535-z

8. Buchman AS, Bennet DA. Cognitive frailty. J Nutr Health Aging. 2013;14(9):738-739. https://doi.org/10.1007/s12603-013-0397-9

9. Kelaiditi E, Cesari M, Canevelli M, et al. Cognitive frailty: rational and definition from an (IANA/IAGG) international consensus group. J Nutr Health Aging. 2013;17(9):726-734. https://doi.org/10.1007/s12603013-\%200367-2

10. Canvelli M, Cesari M. Cognitive frailty: what is still missing? J Nutr Health Aging. 2015;19(3):273-275. https://doi.org/10.1007/s12603015-0464-5

11. Woods AJ, Cohen RA, Pahor M. Cognitive frailty: frontiers and challenges. J Nutr Health Aging. 2013;17(9):741-743. https://doi.org/ 10.1007/s12603-013-0398-8

12. Panza F, Solfrizzi V, Barulli MR, et al. Cognitive frailty: a systematic review of epidemiological and neurobiological evidence of an agerelated clinical condition. Rejuvenation Res. 2015;18(5):389-412. https://doi.org/10.1089/rej.2014.1637

13. Sargent L, Brown R. Assessing the current state of cognitive frailty: measurement properties. J Nutr Health Aging. 2017;21(2):152-160. https://doi.org/10.1007/s12603-016-0735-9

14. Mitchell AJ, Beaumont H, Ferguson D, Yadegarfar M, Stubbs B. Risk of dementia and mild cognitive impairment in older people with subjective memory complaints: meta-analysis. Acta Psychiatr Scand. 2014;130(6): 439-451. https://doi.org/10.1111/acps.12336

15. Luck T, Roehra S, Jessen F, Villringer A, Angermeyer MC, Riedel-Heller SG. Mortality in individuals with subjective cognitive decline: results of the Leipzig longitudinal study of the aged (LEILA75+). J Alzheimers Dis. 2015;48(s1):33-42. https://doi.org/10.3233/JAD-150090

16. Zuniga KE, Mackenzie MJ, Kramer A, McAuley E. Subjective memory impairment and well-being in community-dwelling older adults. Psychogeriatrics. 2016;16(1):20-26. https://doi.org/10.1111/psyg.12 112

17. Mol M, Carpay M, Ramakers I, Rozendaal N, Verhey F, Jolles J. The effect of perceived forgetfulness on quality of life in older adults; a qualitative review. Int J Geriatr Psychiatry. 2007;22(5):393-400. https://doi.org/10.1002/gps.1686

18. Nasreddine ZS, Phillips NA, Bedirian V, et al. The Montreal Cognitive Assessment, MoCA: a brief screening tool for mild cognitive impairment. J Am Geriatr Soc. 2005;53(4):695-699. https://doi.org/10.1111/j.15325415.2005.53221.x

19. Luis CA, Keegan AP, Mullan M. Cross validation of the Montreal Cognitive Assessment in community dwelling older adults residing in the southeastern US. Int J Geriatr Psychiatry. 2009;24(2):197-201. https://doi.org/10.1002/gps.2101

20. Magierska J, Magierski R, Fendler W, Kłoszewska I, Sobów TM. Clinical application of the Polish adaptation of the Montreal Cognitive Assessment (MoCA) test in screening for cognitive impairment. Neurol
Neurochir Pol. 2012;46(2):130-139. https://doi.org/10.5114/ninp.201 2.28255

21. Selekler K, Cangoz B, Uluç S. Power of discrimination of Montreal Cognitive Assessment (MoCA) scale in Turkish patients with mild cognitive impairment and Alzheimer's disease. Turk J Geriatr. 2010;13:166-171.

22. De Jonghe JFM, Schmand B. Informant Vragenlijst over Cognitieve Achteruitgang bij Ouderen; Nederlandse versie (IQCODE-N); 1996.

23. Jorm AF, Jacomb PA. The Informant Questionnaire on Cognitive Decline in the Elderly (IQCODE): socio-demographic correlates, reliability, validity and some norms. Psychol Med. 1989;19(04):1015-1022. https://doi.org/10.1017/S0033291700005742

24. Ehrensperger MM, Berres M, Taylor KI, Monsch AU. Screening properties of the German IQCODE with a two-year time frame in $\mathrm{MCl}$ and early Alzheimer's disease. Int Psychogeriatr. 2010;22(01):91-100. https://doi.org/10.1017/S1041610209990962

25. De Witte N, Campens J, De Donder L, Dierckx E, Rammelaere S, Verté D. 2016. Oudere mensen blijven langer thuis wonen: Zicht krijgen op kwetsbare groepen wordt belangrijk. http://sociaalnet/analyse-xl/ oudere-mensen-blijven-langer-thuis-wonen/ Published November 29 2016. Accessed on May 222017.

26. Mason $\mathrm{CH}$, Perreault WD. Collinearity, power, and interpretation of multiple regression analysis. J Market Res. 1991;28(3):268-280. https://doi.org/10.2307/3172863

27. Montejo P, Montenegro M, Fernández MA, Maestu F. Subjective memory complaints in the elderly: prevalence and influence of temporal orientation, depression and quality of life in a population-based study in the city of Madrid. Aging Ment Health. 2011;15(1):85-96. https:// doi.org/10.1080/13607863.2010.501062

28. Slavin MJ, Brodaty H, Kochan NA, et al. Prevalence and predictors of "subjective cognitive complaints" in the Sydney Memory and Ageing Study. Am J Geriatr Psychiatry. 2010;18(8):701-710. https://doi.org/ 10.1097/JGP.0b013e3181df49fb

29. De Deyn PP, Goeman J, Vervaet A, Dourcy-Belle-Rose B, Van Dam D, Geerts E. Prevalence and incidence of dementia among 7580-year-old community-dwelling elderly in different districts of Antwerp, Belgium: the Antwerp Cognition (ANCOG) Study. Clin Neurol Neurosurg. 2011;113:736-745. org/101016/jclineuro201107030

30. Wilson RS, Sytsma J, Barnes LL, Boyle PA. Anosognosia in dementia. Curr Neurol Neurosci Rep. 2016;16(9):77. https://doi.org/10.1007/ s11910-016-0684-z

\section{SUPPORTING INFORMATION}

Additional Supporting Information may be found online in the supporting information tab for this article.

How to cite this article: De Roeck EE, Dury S, De Witte N, et al. CFAl-Plus: Adding cognitive frailty as a new domain to the comprehensive frailty assessment instrument. Int J Geriatr Psychiatry. 2018;33:941-947. https://doi.org/10.1002/gps.4875 